\title{
Produção de biocompósitos de biopolietileno/farinha de madeira compatibilizados com PE-g-MA. Avaliação do teor de anidrido maleico nas propriedades mecânicas e termomecânicas
}

Production of bio-polyethylene/wood flour biocomposites compatible with PE-g-MA. Assessment of maleic anhydride content in mechanical and thermomechanical properties

Producción de biocompuestos de biopolietileno/harina de madera compatibles con PE-g-MA. Evaluación del contenido de anhídrido maleico en propiedades mecánicas y termomecánicas

Recebido: 19/05/2021 | Revisado: 28/05/2021 | Aceito: 05/06/2021 | Publicado: 20/06/2021

Fabiano Santana da Silva ORCID: https://orcid.org/0000-0002-4808-7112 Universidade Estadual da Paraíba, Brasil

E-mail: sunsolaris8@hotmail.com

Wallisson Alves da Silva

ORCID: https://orcid.org/0000-0002-0281-4812 Universidade Federal de Campina Grande, Brasil E-mail: wallissonalves11@hotmail.com

Carlos Bruno Barreto Luna

ORCID: https://orcid.org/0000-0002-2441-7439 Universidade Federal de Campina Grande, Brasil

E-mail: brunobarretodemaufcg@ hotmail.com

Eduardo da Silva Barbosa Ferreira ORCID: https://orcid.org/0000-0002-2670-2794 Universidade Federal de Campina Grande, Brasil E-mail: eduardosbf95@gmail.com

Edcleide Maria Araújo ORCID: https://orcid.org/0000-0003-4906-864X Universidade Federal de Campina Grande, Brasil E-mail: edcleide.araujo@ufcg.edu.br

\begin{abstract}
Resumo
A produção de materiais ecológicos está sendo incentivada, visando minimizar os impactos ambientais e promover uma maior sustentabilidade. Portanto, esse trabalho, teve como objetivo desenvolver biocompósitos de biopolietileno (BioPE)/farinha de madeira (FM), utilizando como compatibilizante o polietileno enxertado com anidrido maleico (PE-g-MA). Os biocompósitos foram preparados em uma extrusora de rosca dupla corrotacional e moldados por injeção. As propriedades de resistência ao impacto Izod, resistência à tração, Dureza Shore D, Temperatura de Deflexão Térmica (HDT) e absorção de água foram investigadas. A resistência ao impacto aumentou quando o biocompósito BioPE/FM foi compatibilizado com PE-g-MA. Esse acréscimo foi mais expressivo para 10\% de PE-gMA com alto grau de enxertia de anidrido maleico, indicando um maior nível de interação entre as fases. Os biocompósitos BioPE/FM/PE-g-MA apresentaram propriedades de módulo elástico, resistência à tração, dureza Shore D e HDT aprimoradas, em comparação ao biocompósito não compatibilizado. Um aspecto importante foi a redução da absorção de água para os biocompósitos compatibilizados com PE-g-MA, sugerindo um maior efeito barreira para a difusão de umidade. Do ponto de vista do grau de enxertia de anidrido maleico no PE-g-MA, em geral, a resistência ao impacto foi a propriedade mais sensível. Os resultados indicam que a farinha de madeira é um resíduo com potencial para ser reaproveitado no desenvolvimento de biocompósitos.
\end{abstract}

Palavras-chave: Reaproveitamento; Farinha de madeira; Biocompósitos; Compatibilizante.

\begin{abstract}
The production of ecological materials is being encouraged, aiming to minimize environmental impacts and promote greater sustainability. Therefore, this work aimed to develop bio-polyethylene biocomposites (BioPE)/wood flour (WF), using polyethylene grafted with maleic anhydride (PE-g-MA) as a compatibilizer. The biocomposites were prepared in a corrotational twin screw extruder and injection molded. The properties of Izod impact strength, tensile strength, Shore D hardness, Heat Deflection Temperature (HDT) and water absorption were investigated. Impact strength improved when biocomposite BioPE/WF was made compatible with PE-g-MA. This increase was more expressive for $10 \%$ of PE-g-MA with a high degree of maleic anhydride grafting, indicating a higher level of
\end{abstract}


interaction between the phases. The BioPE/WF/PE-g-MA biocomposites showed improved elastic modulus, tensile strength, Shore D and HDT hardness, compared to the non-compatible biocomposite. An important aspect was the reduction of water absorption for biocomposites compatible with PE-g-MA, suggesting a greater barrier effect for the diffusion of moisture. From the point of view of the degree of grafting of maleic anhydride in PE-g-MA, in general, impact strength was the most sensitive property. The results indicate that wood flour is a waste with the potential to be reused in the development of biocomposites.

Keywords: Reuse; Wood flour; Biocomposites; Compatibilizer.

\section{Resumen}

Se fomenta la producción de materiales ecológicos, con el objetivo de minimizar los impactos ambientales y promover una mayor sostenibilidad. Por lo tanto, este trabajo tuvo como objetivo desarrollar biocompuestos de biopolietileno (BioPE)/harina de madera (HM), utilizando polietileno injertado con anhídrido maleico (PE-g-MA) como compatibilizador. Los biocomposites se prepararon en una extrusora de doble husillo rotacional y se moldearon por inyección. Se investigaron las propiedades de resistencia al impacto Izod, resistencia a la tracción, dureza Shore $\mathrm{D}$, temperatura de deflexión térmica (HDT) y absorción de agua. La resistencia al impacto aumentó cuando el biocompuesto BioPE/HM se hizo compatible con PE-g-MA. Este aumento fue más expresivo para el 10\% de PE-gMA con un alto grado de injerto de anhídrido maleico, lo que indica un mayor nivel de interacción entre las fases. Los biocompuestos BioPE/HM/PE-g-MA mostraron propiedades mejoradas de módulo elástico, resistencia a la tracción, dureza Shore D y HDT, en comparación con el biocompuesto no compatible. Un aspecto importante fue la reducción de la absorción de agua para biocomposites compatibles con PE-g-MA, sugiriendo un mayor efecto barrera para la difusión de la humedad. Desde el punto de vista del grado de injerto de anhídrido maleico en PE-g-MA, en general, la resistencia al impacto fue la propiedad más sensible. Los resultados indican que la harina de madera es un residuo con potencial de reutilización en el desarrollo de biocomposites.

Palabras clave: Reutilización; Harina de madera; Biocomposites; Compatibilizador.

\section{Introdução}

Os biocompósitos são materiais produzidos a partir de polímeros derivados de recursos renováveis e fibras naturais (Nguyen et al., 2020; Tarrés et al., 2021; Siqueira et al., 2021). Do ponto de vista ambiental, os biocompósitos chamaram atenção da indústria e da comunidade científica, uma vez que apresentam boas propriedades mecânicas e são ecológicos (Lee et al., 2020; Siqueira et al., 2020). Esses novos materiais verdes receberam interesses significativos nos últimos anos, devido a nova legislação que incentiva tecnologias de manufaturas sustentáveis (Silva et al., 2021; Tarrés e Ardanuy, 2020). Nesse contexto, os biocompósitos com matriz polimérica de biopolietileno (BioPE), policaprolactona (PCL) e poli(acido lático) (PLA) estão sendo bastante explorados, utilizando as fibras naturais como cargas de reforço (Achla e Jacob, 2018; Immonem et al., 2019; Siqueira et al., 2019; Bazan et al., 2020a; Morais et al., 2019).

As vantagens da utilização de fibras naturais para produção de biocompósitos são justificadas pelo custo relativamente baixo, benefícios sociais, materiais ecológicos e boas propriedades mecânicas (Kotik, 2019). As fibras naturais são totalmente biodegradáveis e são derivadas de recursos renováveis, com boas características bioquímicas, especialmente por serem atóxicas e não cancerígenas (Bazan et al., 2020b). Existem muitas fibras vegetais disponíveis para a produção de biocompósitos, com destaque para: curauá, dendê, farinha de madeira, palha de arroz, bambu, sisal, cânhamo, bagaço da canade-açucar, folha de abacaxi, palha de trigo, algodão, linho, casca de arroz, coco, kenaf, banana, milho e juta (Jesus et al., 2019). O principal problema da produção de biocompósitos de base vegetal, em geral, é a incompatibilidade entre as fibras naturais e a matriz polimérica, impactando em uma baixa adesão interfacial entre os dois componentes e, como consequência, gerando baixas propriedades mecânicas (Raia et al., 2021; Ferreira et al., 2020; Wearn et al., 2020). Para contornar esse problema, a incorporação de um agente compatibilizante torna-se essencial para melhorar a adesão entre o polímero e a fibra natural (Luna et al., 2019). O agente de compatibilização mais utilizado em compósitos poliméricos com fibras naturais consiste em polímero modificado com anidrido maleico (MA), como, por exemplo, o polietileno enxertado com anidrido maleico (PE-g-MA) (Poletto, 2017). Esse compatibilizante é adequado para biocompósitos com matriz polimérica de biopolietileno com fibras naturais, uma vez que sua estrutura apresenta uma parte apolar semelhante à matriz polimérica e uma parte polar que reage 
com os grupos funcionais das fibras naturais.

O biopolietileno (BioPE) é produzido a partir do monômero de etileno obtido por meio da desidratação do etanol baseado na cana-de-açúcar e, portanto, proveniente de uma fonte renovável (Hanken et al., 2019). Porém, o BioPE não é biodegradável, ou seja, apresenta uma durabilidade semelhante ao polietileno convencional. Além disso, as características químicas, mecânicas e de processabilidade do BioPE são semelhantes ao polietileno de origem petroquímica (Bezerra et al., 2017). O argumento para o BioPE ser considerado um material ecológico está baseado em manter o equilíbrio do dióxido de carbono $\left(\mathrm{CO}_{2}\right)$, uma vez que o $\mathrm{CO}_{2}$ capturado da atmosfera pela biomassa, quando liberado na atmosfera por combustão, é novamente capturado pela cana-de-açúcar pelo processo de fotossíntese (Bezerra et al., 2019). Em vista disso, o BioPE é um biopolímero atraente para produção de biocompósitos com fibras naturais, tendo em vista as boas características mecânicas, térmicas e ambientais (Castro et al., 2013). Alguns trabalhos na literatura (Castro et al., 2012; Castro et al., 2017; Tarrés et al., 2018; Dominici et al., 2019; Espinach et al., 2020) foram desenvolvidos com foco na preparação de biocompósitos de biopolietileno/fibras naturais, gerando materiais ecológicos com boas propriedades mecânicas. Entretanto, no que se refere ao desenvolvimento de biocompósitos de BioPE com a farinha de madeira, há uma escassez de trabalhos na literatura. Portanto, torna-se interessante avaliar o sistema BioPE/farinha de madeira, uma vez que essa fibra é descartada em grandes quantidades das indústrias madeireiras. O reaproveitamento é incentivado devido ao fato de poder agregar valor a um material que seria descartado e, ao mesmo tempo, minimizar o impacto ambiental e social (Luna et al., 2015).

Diante do exposto, o presente trabalho, teve como objetivo avaliar as propriedades mecânicas e termomecânicas de biocompósitos de biopolietileno (BioPE)/farinha de madeira (FM), utilizando o polietileno enxertado com anidrido maleico (PE-g-MA) como compatibilizante. O impacto do teor de anidrido maleico no PE-g-MA foi avaliado na compatibilização dos biocompósitos BioPE/FM.

\section{Metodologia}

\section{Materiais}

- O polietileno de alta densidade verde, biopolietileno (BioPE), comercializado com o código SHC7260® e índice de fluidez (IF) de 7,2 g/10 $\min \left(190^{\circ} \mathrm{C} / 2,16 \mathrm{~kg}\right)$, produzido a partir da cana-de-açúcar e fornecido na forma de pellets pela Braskem.

- Utilizou-se como carga a farinha de madeira (FM) de maçaranduba (40 mesh), com nome científico Manilkara spp, proveniente da indústria madeireira, sediada na cidade de Campina Grande/PB, Brasil.

- O polietileno enxertado com anidrido maleico (PE-g-MA) foi utilizado como agente compatibilizante, comercializado com o código $3029 \circledR$ e contendo de 1,5 a 1,7\% de anidrido maleico (MA), fornecido na forma de pellets pela Addivant. Densidade de 0,95 g/cm3 e IF = 4,0 g/10 min $\left(190^{\circ} \mathrm{C} / 2,16 \mathrm{~kg}\right)$.

- O polietileno enxertado com anidrido maleico (PE-g-MA) foi utilizado como agente compatibilizante, comercializado com o código $3009 \circledast$ e contendo de 0,8 a 1,2\% de anidrido maleico (MA), fornecido na forma de pellets pela Addivant. Densidade de $0,95 \mathrm{~g} / \mathrm{cm} 3 \mathrm{e} \mathrm{IF}=5,0 \mathrm{~g} / 10 \mathrm{~min}\left(190^{\circ} \mathrm{C} / 2,16 \mathrm{~kg}\right)$.

\section{Métodos}

\section{Preparação dos concentrados}

Os concentrados poliméricos foram preparados pelo método de intercalação por fusão, usando um misturador de alta rotação (Homogeneizador Termocinético), modelo $\mathrm{MH}-50 \mathrm{H}$ da marca $\mathrm{MH}$ equipamentos. O equipamento foi acionado durante um período de aproximadamente 10 segundos, ocorrendo à mistura e a fusão do material por atrito. Após o término deste procedimento, o material foi triturado em moinho de facas. A Figura 1 apresenta o processo de obtenção dos concentrados de 
BioPE/farinha de madeira.

Figura 1. Processo de obtenção dos concentrados poliméricos.

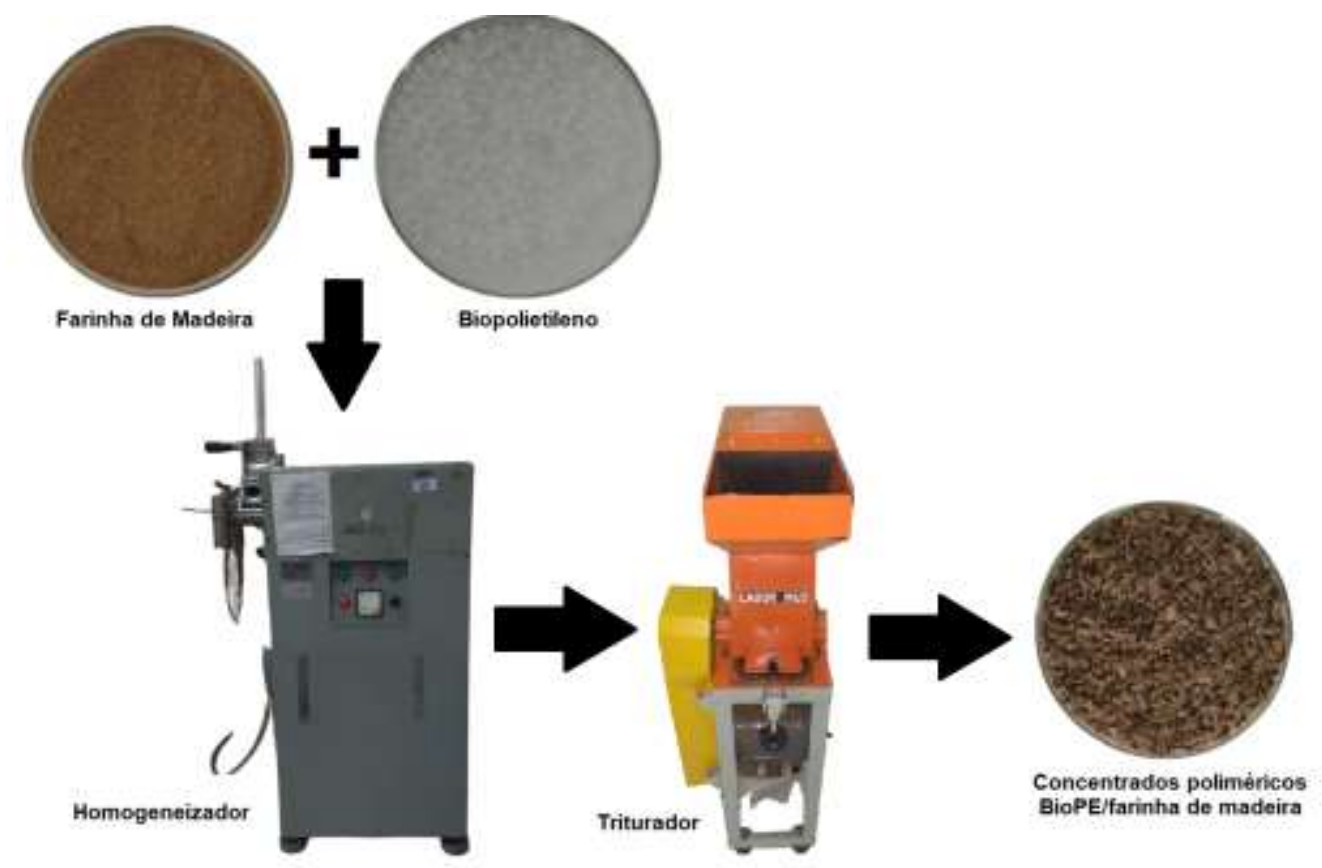

Fonte: Autores.

\section{Processamento por Extrusão}

A Tabela 1 ilustra as proporções em massa $(\%)$ das composições que foram utilizadas no desenvolvimento dos biocompósitos.

Tabela 1. Composições dos biocompósitos com as proporções em massa (\%). (A)*: alto grau de enxertia $(1,5$ a $1,7 \%$ de anidrido maleico); (B)**: baixo grau de enxertia ( 0,8 a $1,2 \%$ de anidrido maleico).

\begin{tabular}{l|c|c|c|c}
\hline \multicolumn{1}{c|}{ Amostras } & $\begin{array}{c}\text { BioPE } \\
(\% \text { em massa })\end{array}$ & $\begin{array}{c}\text { FM } \\
(\% \text { em massa })\end{array}$ & $\begin{array}{c}\text { PE-g-MA (A)* } \\
(\% \text { em massa) }\end{array}$ & $\begin{array}{c}\text { PE-g-MA 2 (B) } \\
(\% \text { em massa) }\end{array}$ \\
\hline BioPe/FM & 80 & 20 & - & - \\
BioPe/FM/PE-g-MA & 75 & 20 & 5 & - \\
BioPe/FM/PE-g-MA & 70 & 20 & 10 & - \\
BioPe/FM/PE-g-MA & 75 & 20 & - & 5 \\
BioPe/FM/PE-g-MA & 70 & 20 & - & 10 \\
\hline
\end{tabular}

Fonte: Autores.

Os biocompósitos foram misturados a seco e, posteriormente, processados em uma extrusora de rosca dupla corrotacional modular, modelo ZSK $(\mathrm{D}=18 \mathrm{~mm}$ e L/D = 40), da Coperion Werner-Pfleiderer, com perfil de temperatura de $160^{\circ} \mathrm{C}, 160^{\circ} \mathrm{C}, 165^{\circ} \mathrm{C}, 165^{\circ} \mathrm{C}, 170^{\circ} \mathrm{C}, 170^{\circ} \mathrm{C}$ e $170^{\circ} \mathrm{C}$, velocidade de rotação da rosca de 250 rpm e taxa de alimentação controlada de $4 \mathrm{~kg} / \mathrm{h}$, com perfil de rosca configurado com elementos de misturas distributivos e dispersivos. Posteriormente, os biocompósitos foram granulados e secos em uma estufa com vácuo durante $24 \mathrm{~h}$, em temperatura de $60^{\circ} \mathrm{C}$.

Os biocompósitos foram moldados por injeção, em uma injetora Arburg, Modelo Allrounder 207C Golden Edition, para a obtenção de corpos de prova de impacto, tração e HDT, segundo as normas ASTM D256, ASTM D638 e ASTM D648, 
respectivamente. As condições de moldagem dos corpos de prova são apresentadas na Tabela 2.

Tabela 2. Condições de moldagem por injeção dos corpos de prova.

\begin{tabular}{c|c}
\hline \multicolumn{2}{c}{ Condições } \\
\hline Pressão de injeção (bar) & 800 \\
Perfil de temperature $\left({ }^{\circ} \mathrm{C}\right)$ & $160,160,165,170,170$ \\
Temperatura do molde $\left({ }^{\circ} \mathrm{C}\right)$ & 20 \\
Tempo de resfriamento no molde (s) & 30 \\
Pressão de recalque (bar) & 500 \\
\hline
\end{tabular}

Fonte: Dados da pesquisa.

A metodologia científica adotada é de natureza quantitativa, tendo em vista que se trabalhou com quantidades prédeterminadas de cada componente, bem como utilizou-se parâmetros de processamento específicos (Lima et al., 2021). Em seguida, os corpos de provas passaram por análises experimentais, sendo obtidos dados numéricos.

\section{Caracterização dos Materiais}

O ensaio de resistência ao impacto Izod foi realizado em corpos de prova entalhados, segundo a norma ASTM D256, em um aparelho da marca Ceast modelo Resil 5,5 J, operando com martelo de 2,75 J, em temperatura ambiente $\left(\sim 23^{\circ} \mathrm{C}\right)$. Os resultados foram analisados com uma média de sete corpos de prova.

O ensaio de tração foi realizado em corpos de prova injetados, segundo a norma ASTM D638, utilizando uma máquina de ensaios universal da marca EMIC DL 2000, com velocidade de $50 \mathrm{~mm} / \mathrm{min}$ e célula de carga de $20 \mathrm{kN}$, em temperatura ambiente $\left(\sim 23^{\circ} \mathrm{C}\right)$. Os resultados foram analisados com uma média de sete corpos de prova.

A determinação da resistência à penetração foi realizada de acordo com a norma ASTM D2240, em equipamento Shore-Durometer Hardness Type "D", com uma carga de $50 \mathrm{~N}$ controlada por molas calibradas por meio de indentores padronizados para o durômetro. Os resultados foram analisados com uma média de sete penetrações.

A temperatura de deflexão térmica (HDT) foi obtida conforme a norma ASTM D648, em um equipamento Ceast, modelo HDT 6 VICAT, com uma tensão de $455 \mathrm{kPa}$ e taxa de aquecimento de $120^{\circ} \mathrm{C} / \mathrm{h}$. A temperatura foi determinada após a amostra ser defletida $0,25 \mathrm{~mm}$. Os resultados foram analisados com uma média de três corpos de prova.

O teste de absorção de água foi baseado no procedimento sugerido na ASTM D570. Os corpos de prova foram condicionados em uma estufa, durante $24 \mathrm{~h} \mathrm{sob} 50^{\circ} \mathrm{C}$. Após este período, foram imediatamente pesados em uma balança de precisão, sendo obtida a massa antes da imersão (M0). Em seguida, os corpos de prova foram submersos em água à temperatura ambiente $\left(\sim 23^{\circ} \mathrm{C}\right)$, de modo que as amostras ficassem completamente imersas, obtendo a massa após imersão (Mf). Em intervalos de tempo pré-determinados, os corpos de prova foram retirados da água e secos, pesados em balança de precisão e novamente recolocados no banho. A porcentagem de absorção de água foi calculada a partir da Equação 1.

Absorção de água $(\%)=\frac{M_{f}-M_{0}}{M_{0}} \times 100 \%$ 


\section{Resultados e Discussão}

\section{Resistência ao impacto}

Os resultados de resistência ao impacto Izod para os biocompósitos estão apresentados na Figura 2. O biocompósito não compatibilizado BioPE/FM apresentou resistência ao impacto na ordem de 46,5 J/m. A adição de 5 e $10 \%$ do compatibilizante PE-g-MA promoveu um aumento na resistência ao impacto, em comparação ao sistema BioPE/FM. Tal comportamento sugere que aumentou a interação entre o BioPE e a farinha de madeira. Becker et al. (2011) desenvolveram compósitos de polipropileno (PP) com fibras de bananeira (FB), utilizando o polipropileno enxertado com anidrido maleico (PP-MA), como compatibilizante. Os autores indicaram que agentes compatibilizantes enxertados com anidrido maleico são eficazes para aumentar a compatibilidade de compósitos com fibras naturais, uma vez que os grupos anidrido maleico favorecem interações com as hidroxilas das fibras lignocelulósicas, gerando aumento na adesão entre as fases e, em geral, melhorando as propriedades dos compósitos.

Figura 2. Resistência ao impacto dos biocompósitos em função do teor dos compatibilizantes.

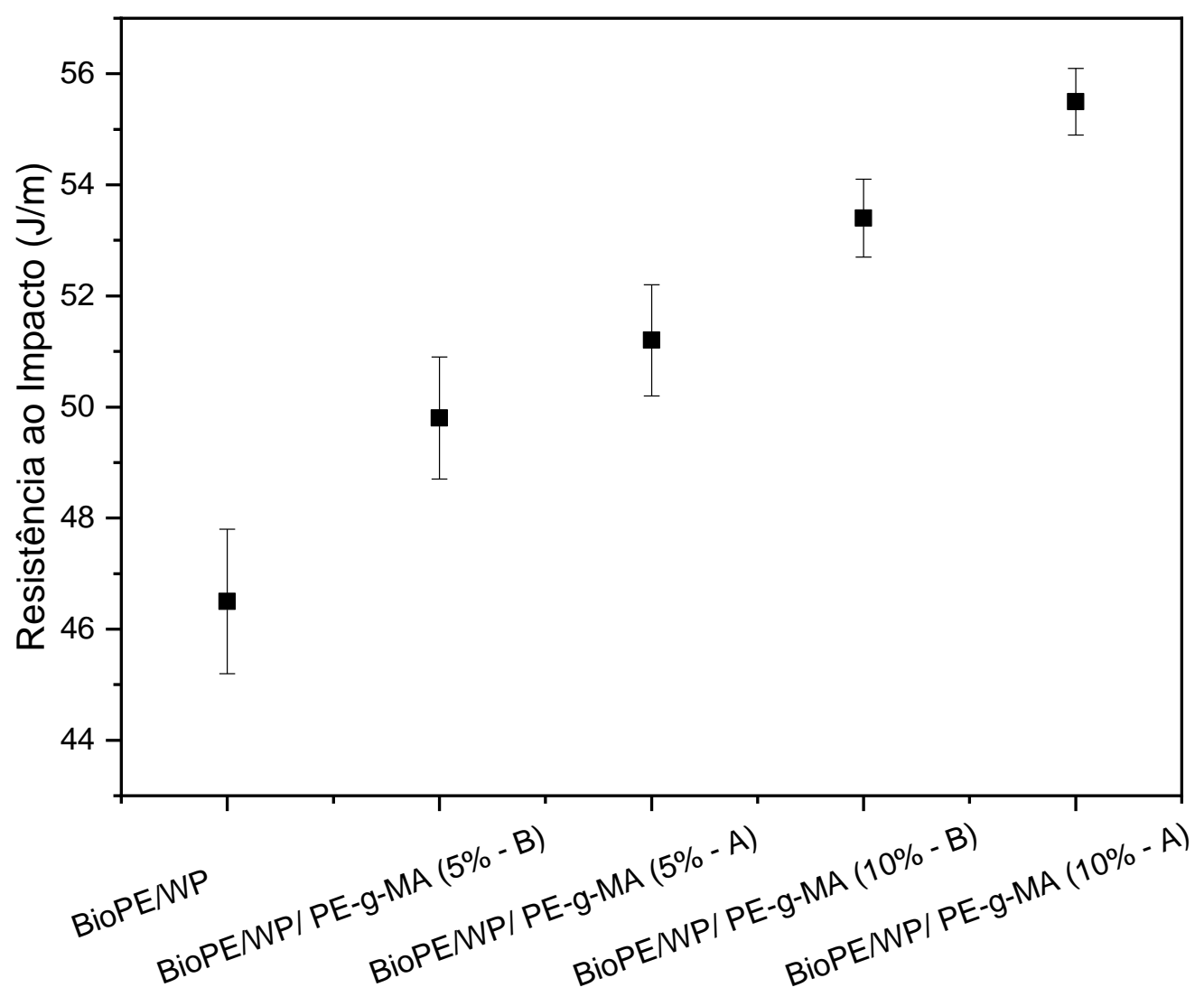

Fonte: Dados da pesquisa.

Os biocompósitos BioPE/FM preparados com 5\% de PE-g-MA, com baixo e alto grau de enxertia de anidrido maleico, mostram que praticamente não ocorreram variações desta propriedade de impacto, uma vez que estão dentro do erro experimental. Contudo, os biocompósitos BioPE/FM/PE-g-MA produzidos com maior concentração de PE-g-MA (10\%) apresentam um aumento mais expressivo na resistência ao impacto, sendo mais pronunciado com alto grau de enxertia de anidrido maleico. O uso de 10\% de PE-g-MA (B) proporcionou ao biocompósito um acréscimo na resistência ao impacto de 
14\% em relação ao sistema BioPE/FM, enquanto que 19\% de ganho foi verificado com a utilização de 10\% de PE-g-MA (A). As composições com concentração de PE-g-MA acima de 5\% apresentam uma tendência de gerar aumentos mais acentuados da resistência ao impacto, devido a maior quantidade de anidrido maleico para reagir com as hidroxilas da farinha de madeira. Ao mesmo tempo, a influência do grau de enxertia foi mais significativa com 10\% de PE-g-MA, indicando que a maior presença de grupos funcionais pode aperfeiçoar o desempenho sob impacto dos biocompósitos BioPE/FM/PE-g-MA.

\section{Propriedades sob Tração}

A Figura 3 apresenta o comportamento do módulo de elasticidade para os biocompósitos, com os diferentes teores de PE-g-MA. O biocompósito BioPE/FM apresentou o menor valor de módulo elástico, indicando uma menor rigidez. A adição de 5\% de PE-g-MA gerou acréscimos no módulo elástico, o que é tomado como um indicativo que o compatibilizante promoveu interação entre as fases presentes, fortalecendo a interface do sistema (Giannetti et al., 2012). Observou-se que, para a composição de 5\% de PE-g-MA, com alto e baixo grau de enxertia, não há diferenças estatisticamente significativas entre as mesmas composições. Quando o teor de PE-g-MA passou para 10\%, o aumento do módulo elástico dos biocompósitos é mais evidente, indicando uma maior compatibilidade. O biocompósito BioPE/FM/PE-g-MA (10\%) com alto grau de enxertia apresentou uma tendência de valores superiores no módulo elástico, em função do seu maior teor do grupo funcional anidrido maleico.

Figura 3. Comportamento do módulo elástico dos biocompósitos, em função do teor do compatibilizante.

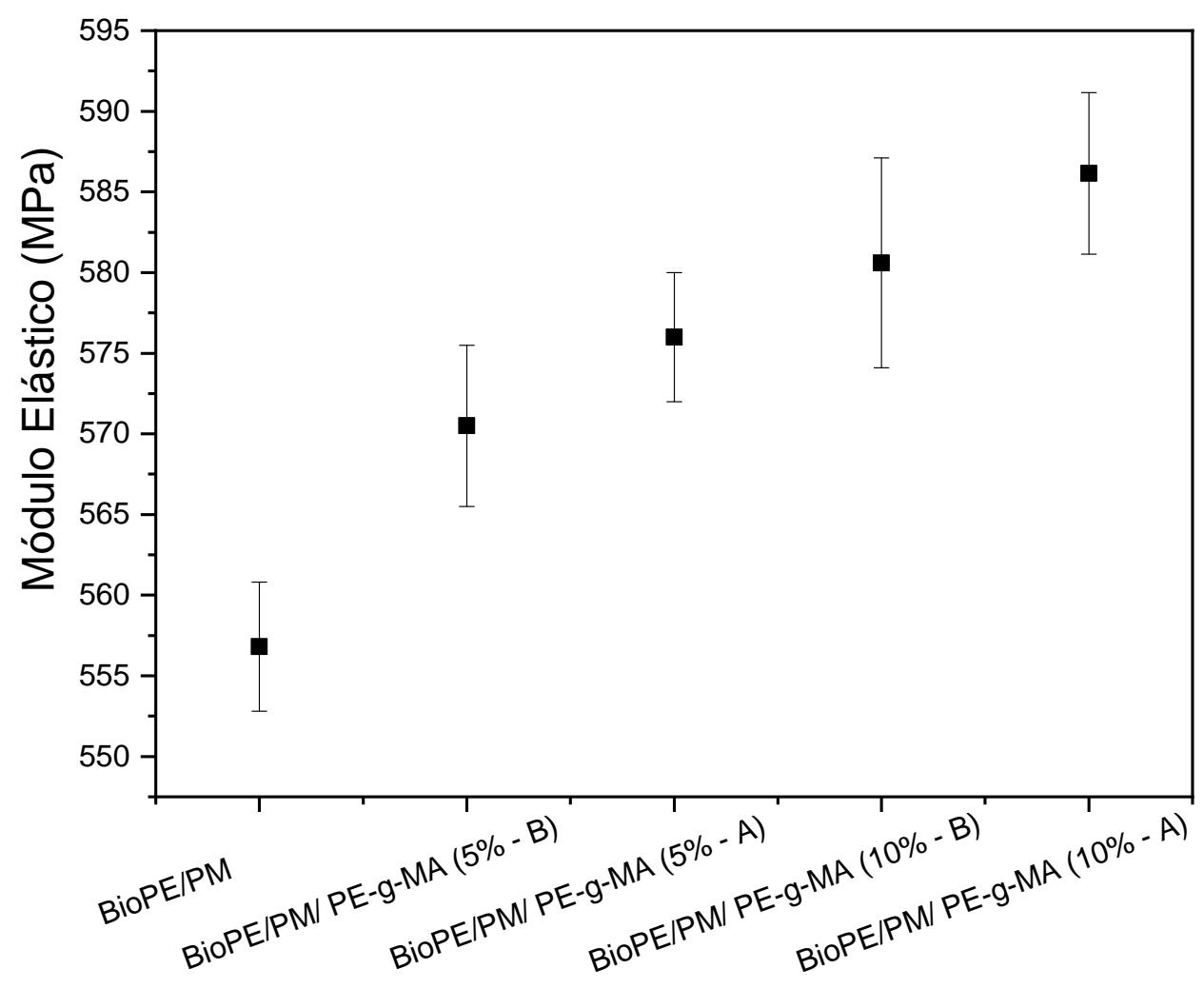

Fonte: Dados da pesquisa.

A Figura 4 apresenta os resultados de resistência à tração do sistema BioPE/FM e dos biocompósitos BioPE/FM/PEg-MA, em função do teor de compatibilizante. A mistura direta de BioPE/FM produziu um biocompósito com resistência à 
tração na ordem de 18,7 MPa. Quando o compatibilizante PE-g-MA foi adicionado, em geral, provocou um aumento em torno de 39\%, em comparação ao sistema BioPE/FM. Estes dados fortalecem as suposições que o PE-g-MA atua como um agente compatibilizante neste sistema BioPE/FM. A compatibilização pode ser atribuída a maior interação promovida entre anidrido maleico do PE-g-MA com as hidroxilas da farinha de madeira, como também verificado por vários autores (Rodrigues et al., 2013; Poletto, 2017b; Wang et al., 2020). Em relação a influência do teor de PE-g-MA, o comportamento da resistência à tração é semelhante para todos os biocompósitos compatibilizados.

Figura 4. Resistência à tração dos biocompósitos, em função do teor do compatibilizante.

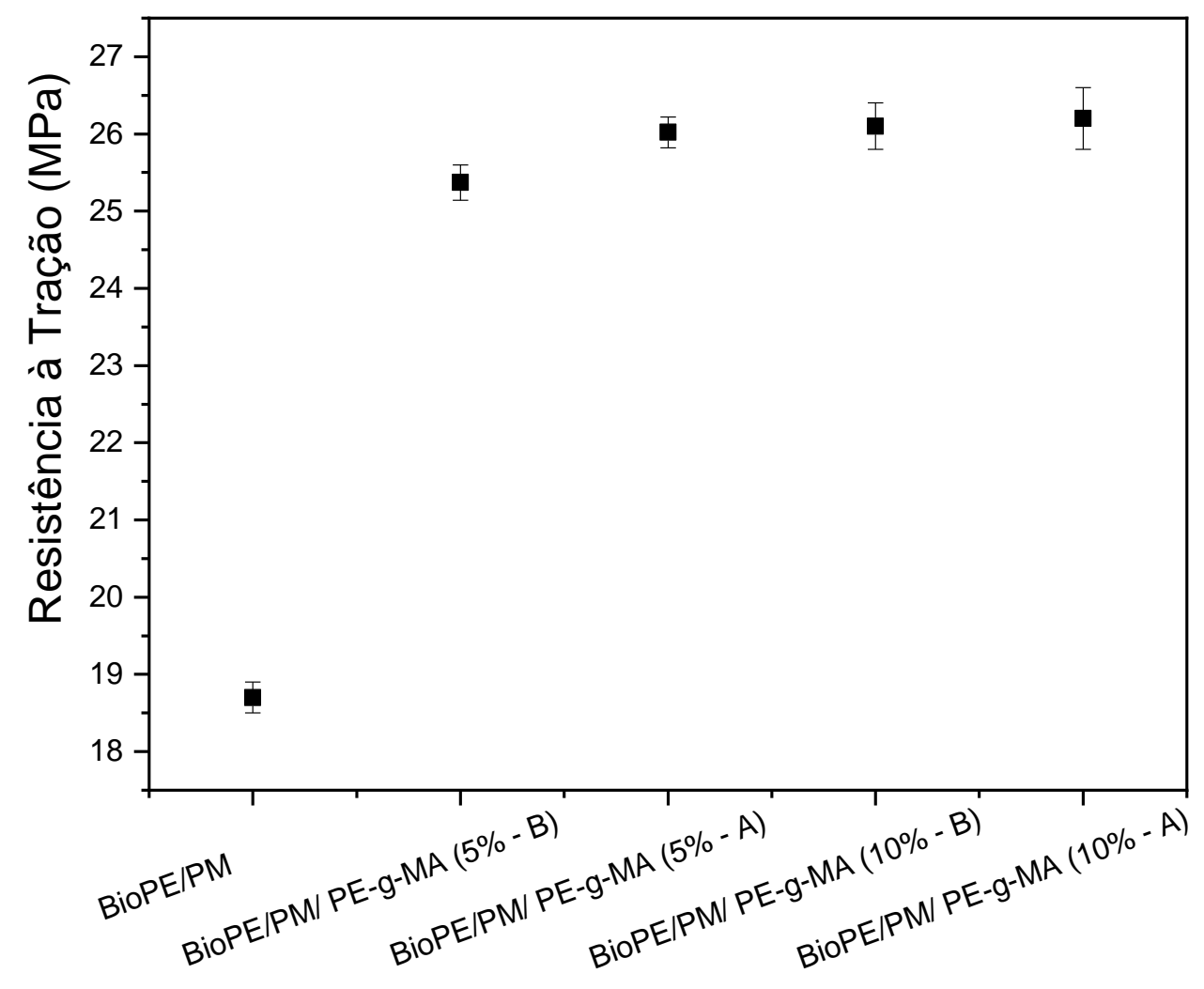

Fonte: Dados da pesquisa.

\section{Dureza Shore D}

Os resultados da dureza Shore D dos biocompósitos BioPE/FM e BioPE/FM/PE-g-MA estão apresentados na Figura 5. Essa propriedade mecânica é importante para os biocompósitos, uma vez que avalia a resistência superficial a penetração (Luna et al., 2019b). Observou-se que, o biocompósito não compatibilizado tem uma dureza Shore D em torno de 64,4. Quando o compatibilizante PE-g-MA foi adicionado ao sistema BioPE/FM, independentemente da concentração de compatibilizante, os resultados da dureza Shore D ficaram em torno de 64.8. Nesse caso, considerando a margem de erro experimental, obtiveram-se propriedades de dureza Shore D comparáveis entre si. Aparentemente, a adição do compatibilizante PE-g-MA não afetou essa propriedade de dureza, embora tenha aumentado a concentração de PE-g-MA e o grau de enxertia. Como a dureza Shore D é dependente da característica superficial da amostra, o fator dominante possivelmente foi o teor de farinha de madeira, uma vez que foi mantido $20 \%$ em peso e, por consequência, não houve alteração drástica na dureza Shore D. 
Figura 5. Dureza Shore D dos biocompósitos com 5 e 10\% de PE-g-MA.

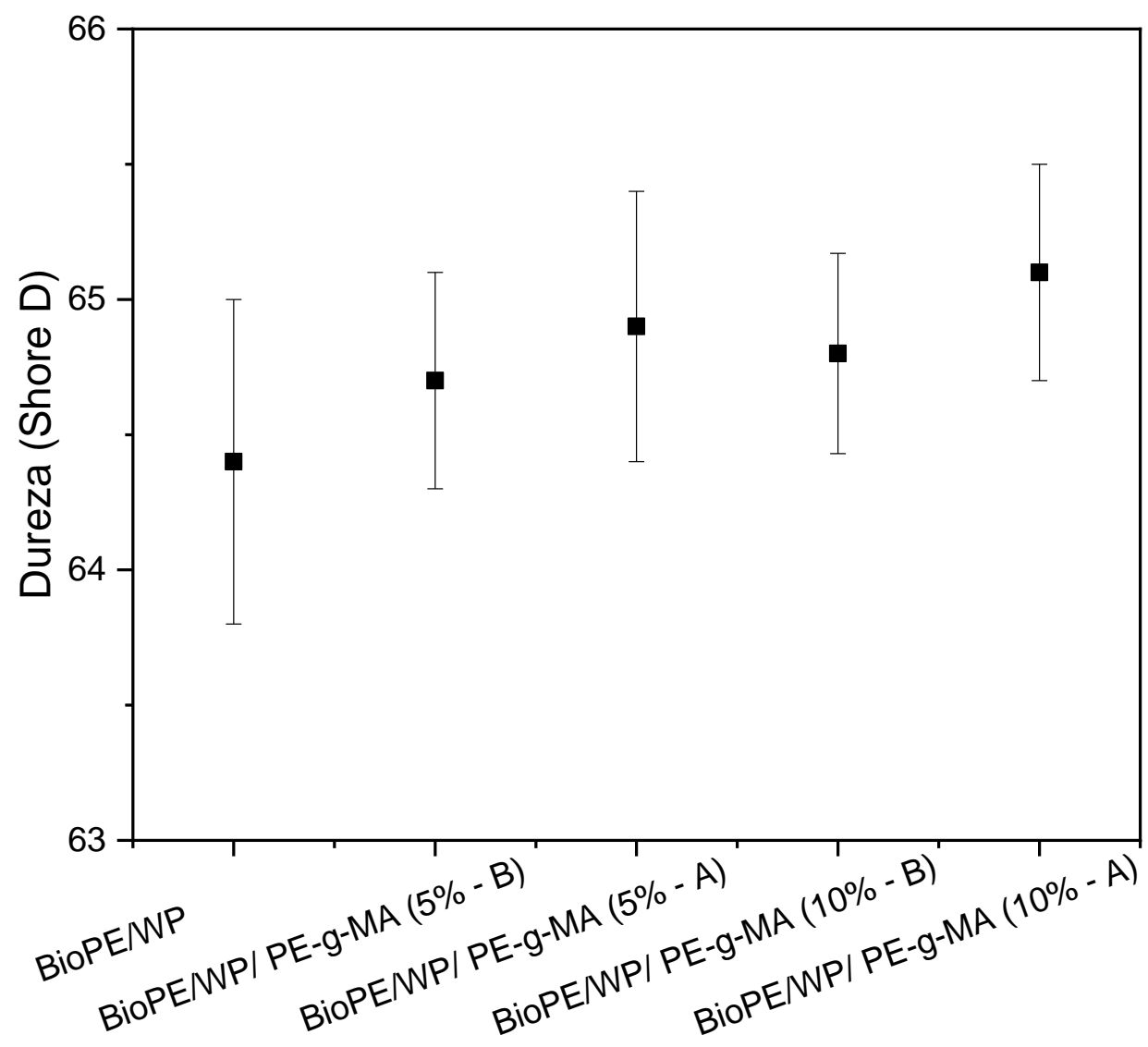

Fonte: Dados da pesquisa.

\section{Temperatura de Deflexão Térmica (HDT)}

A análise termomecânica pode ser utilizada para avaliar a compatibilidade de compósitos poliméricos de forma semelhante às propriedades mecânicas (Silva et al., 2016; Ferreira et al., 2019). A temperatura de deflexão térmica (HDT) é uma propriedade importante para a área da tecnologia de polímeros, uma vez que mensura a estabilidade estrutural do material em temperatura acima da ambiente (Marinho et al., 2020; Luna et al., 2019c).

A Figura 6 apresenta os resultados da temperatura de deflexão térmica (HDT) dos biocompósitos, em função da concentração de PE-g-MA. O biocompósito BioPE/FM apresentou uma HDT na ordem de 83,5 C. A compatibilização do sistema BioPE/FM com 5\% de PE-g-MA provocou um discreto aumento na HDT, em comparação com BioPE/FM não compatibilizado. Não é observado nenhum acréscimo significativo com o aumento do grau de enxertia, uma vez que os resultados são bem próximos. Com uma concentração mais alta de PE-g-MA (10\%), os biocompósitos aumentaram a HDT, sugerindo uma maior estabilidade estrutural em temperaturas mais elevadas. Aparentemente, o maior grau de enxertia do PE-gMA não impactou essa propriedade de HDT, tendo em vista que não há diferenças significativas nos resultados. A HDT é uma propriedade que depende do módulo elástico e do grau de cristalinidade (Luna et al., 2020). Como o módulo elástico aumentou, como verificado na Figura 2, torna-se razoável sugerir que contribuiu para o acréscimo na HDT. 
Figura 6. Temperatura de deflexão térmica dos biocompósitos, em função do teor dos compatibilizantes PE-g-MA.

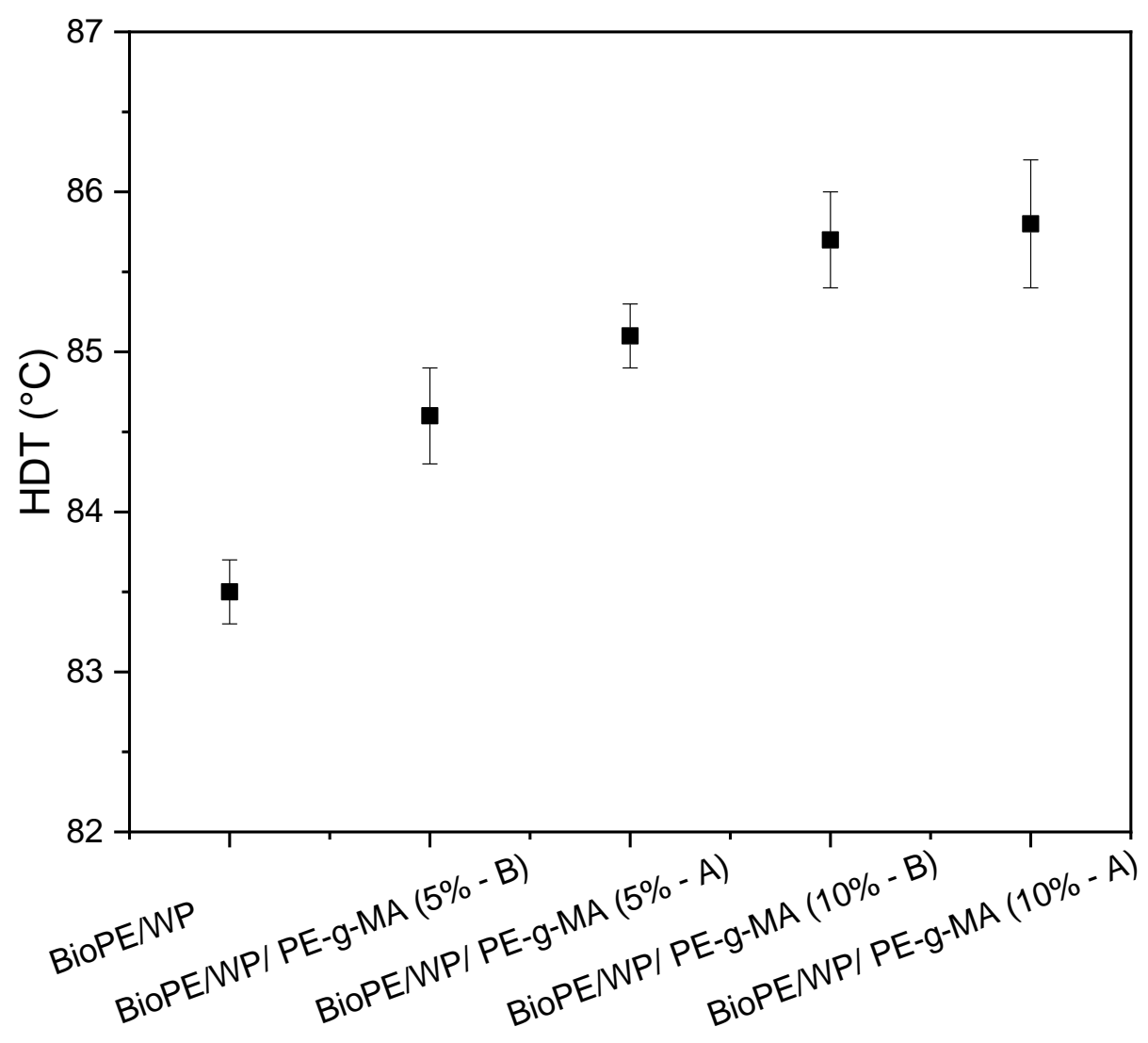

Fonte: Dados da pesquisa.

\section{Absorção de Água}

A Figura 7 mostra o comportamento dos biocompósitos quando submetidos ao teste de absorção de água. O grau de absorção de água é uma importante característica a ser avaliada nos compósitos com fibras naturais, pois avalia o potencial desses materiais para utilização em certas aplicações em contato com umidade (Zahari et al., 2015). Os biocompósitos apresentaram uma rápida cinética de absorção de água até 200 horas de imersão, devido a farinha de madeira. Em geral, as fibras naturais apresentam comportamento hidrofílico, devido à presença dos grupos hidroxilas na estrutura e, portanto, absorvem umidade (Bezerra et al., 2017). Para tempo superior às 200h, o formato da curva vai mudando sua inclinação suavemente até atingir um patamar linear, o qual representa o nível de saturação da umidade. Observou-se que, o biocompósito BioPE/FM apresentou o maior nível de absorção de água, devido possivelmente a defeitos resultantes da baixa adesão entre o BioPE e a farinha de madeira. Porém, quando o compósito BioPE/FM foi compatibilizado com PE-g-MA, houve uma redução significativa na taxa de absorção de água, sugerindo que o compatibilizante aprimorou o efeito barreira, reduzindo a difusão de água para o interior dos biocompósitos. 
Figura 7. Absorção de água do BioPE e dos compósitos, com e sem óleo de macaíba, respectivamente.

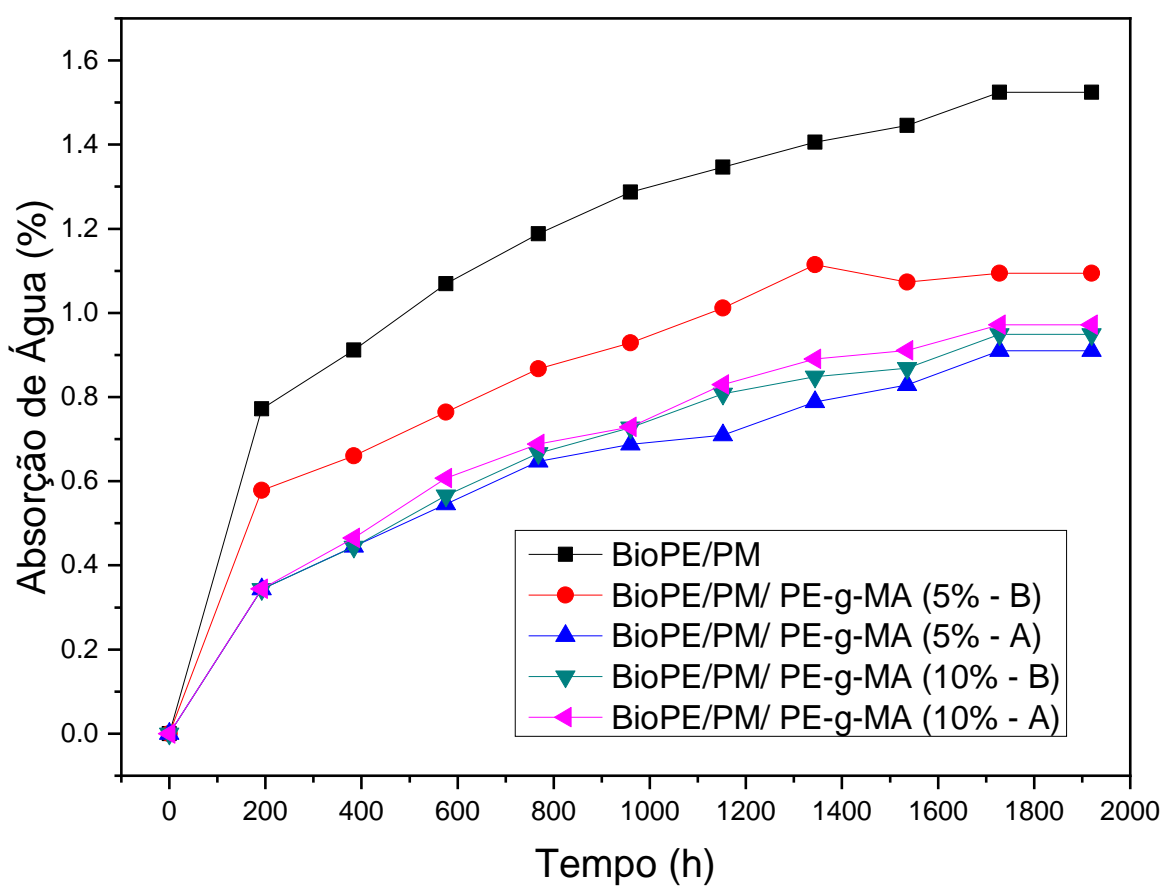

Fonte: Dados da pesquisa.

O biocompósito BioPE/FM/PE-g-MA (5\%) com baixo grau de enxertia apresentou maior absorção de água, em comparação com os demais compostos compatibilizados. Tal comportamento pode ser devido a menor quantidade de anidrido maleico para reagir com as hidroxilas. Contudo, quando o teor de PE-g-MA é 5\% e apresentou alto grau de enxertia, o efeito barreira foi aperfeiçoado, uma vez que reduziu a difusão de umidade, inclusive tendo desempenho similar aos biocompósitos com 10\% em peso de PE-g-MA. Como há interação entre o anidrido maleico e as hidroxilas, torna-se razoável sugerir que houve uma diminuição na disponibilidade dos sítios hidrofílicos na farinha de madeira, impactando na redução da absorção de umidade.

\section{Conclusões}

A influência do PE-g-MA na compatibilização de biocompósitos BioPE/farinha de madeira (FM) foi investigada, utilizando compatibilizante com alto e baixo grau de enxertia de anidrido maleico. Observou-se que, a adição de compatibilizante ao sistema BioPE/FM promoveu um maior desempenho mecânico sob impacto, resistência à tração e módulo elástico, em comparação ao biocompósito BioPE/FM. Contudo, a dureza Shore D não foi modificada com a presença do PE-gMA, uma vez que é uma propriedade dependente da característica superficial. Essa propriedade foi influenciada pelo teor de farinha de madeira ( $20 \%$ em peso), como foi mantida fixa essa concentração, a dureza não foi alterada. Do ponto de vista da resistência termomecânica, a HDT dos biocompósitos compatibilizados, em geral, foi superior ao sistema BioPE/FM sem compatibilizante. Nesse caso, a adição do compatibilizante PE-g-MA contribuiu para aumentar a interação do sistema BioPE/FM, gerando acréscimo na resistência termomecânica e amentando a estabilidade estrutural. Um aspecto importante foi a redução da absorção de umidade dos biocompósitos BioPE/FM/PE-g-MA, sugerindo uma maior resistência a difusão de umidade, em comparação ao biocompósito BioPE/FM. Em relação a influência do grau de enxertia de anidrido maleico no compatibilizante, a resistência ao impacto foi a propriedade mais afetada, com ganhos mais altos com alto grau de enxertia e 
maior concentração de compatibilizante. Os resultados evidenciam que a farinha de madeira é uma fibra natural com potencial para o desenvolvimento de biocompósitos, com boas propriedades. Portanto, a produção de materiais ecológicos é uma maneira de reaproveitar rejeitos da indústria madeireira, o que pode reduzir a quantidade de resíduos sobre o meio ambiente da região e valorizar um material descartado, com possibilidade de reintrodução na cadeia produtiva.

\section{Agradecimentos}

O presente trabalho foi realizado com apoio da Coordenação de Aperfeiçoamento de Pessoal de Nível Superior Brasil (CAPES) - Código de Financiamento 001. Ao mesmo tempo, os autores agradecem à UFCG pela infraestrutura dos laboratórios, ao MCTI/CNPq pelo apoio financeiro e a Addivant pela doação do compatibilizante (PE-g-MA).

\section{Referências}

Achla, S. N. M., \& Jacob, J. (2018). Post-yield fracture correlations to morphological and micromechanical response of poly( $\varepsilon$-caprolactone)-based biocomposites. Journal of Thermoplastic Composite Materials, 31(5), 575-597.

Bazan, P., Mierzwinski, D., Bogucki, R., \& Kuciel, S. (2020a). Bio-Based Polyethylene Composites with Natural Fiber: Mechanical, Thermal, and Ageing Properties. Materials, 13(11), 2595.

Bazan, P., Nosal, P., Kozub, B., \& Kuciel, S. (2020b). Biobased Polyethylene Hybrid Composites with Natural Fiber: Mechanical, Thermal Properties, and Micromechanics. Materials, 13(13), 2967.

Bezerra, E. B., França, D. C., Morais, D. D. S., Fereira, E. S. B., Araújo, E. M., \& Wellen, R. M. R. (2017). Comportamento reológico do Bio-PE e do PCL na presença do PEgAA e PEgMA. Matéria (Rio J.), 22(1), e11798.

Bezerra, A. F. C., Carvalho, L. H., Cavalcanti, W. S., \& Barbosa, A. G. (2016). Mechanical behavior of composites reinforced with fibers caroa. Fibers and Polymers, 17(1), 1908-1915.

Becker, D., Kleinschmidt, A. C., Balzer, P. S., \& Soldi, V. (2011). Influência da Sequência de Mistura do PP-MA nas Propriedades dos Compósitos de PP e Fibra de Bananeira. Polímeros, 21(1): 7-12.

Bezerra, E. B., França, D. C., Morais, D. D. S., Siqueira, D. D., Araújo, E. M., \& Wellen, R. M. R. (2019). Toughening of bio-PE upon addition of PCL and PEgAA. REM - International Engineering Journal, 72(3), 469-478.

Castro, D. O., Frollini, E., Marini, J., \& Filho, A. R. (2013). Preparação e Caracterização de Biocompósitos Baseados em Fibra de Curauá, Biopolietileno de Alta Densidade (BPEAD) e Polibutadieno Líquido Hidroxilado (PBHL). Polímeros, 23(1): 65-73.

Castro, D. O., Filho, A. R., \& Frollini, E. (2012). Materials prepared from biopolyethylene and curaua fibers: Composites from biomass. Polymer Testing, $31(7), 880-888$

Castro, D. O., Passado, F. R., Filho, A. R., \& Frollini, E. (2017). Use of castor and canola oils in "biopolyethylene" curauá fiber composites. Composites Part A: Applied Science and Manufacturing, 95(4), 22-30.

Dominici, F., Garcia, D. G., Fombuena, V., Luzi, F., Puglia, D., Torre, L., \& Balart, R. (2019). Bio-Polyethylene-Based Composites Reinforced with Alkali and Palmitoyl Chloride-Treated Coffee Silverskin. Molecules, 24(17), 3113.

Espinach, F. X., Espinosa, E., Reixach, R., Rodriguez, A., Mutjé, P., \& Tarrés, Q. (2020). Study on the Macro and Micromechanics Tensile Strength Properties of Orange Tree Pruning Fiber as Sustainable Reinforcement on Bio-Polyethylene Compared to Oil-Derived Polymers and Its Composites. Polymers, 12(10), 2206.

Ferreira, E. S. S., Luna, C. B. B., Araújo, E. M., Siqueira, D. D., \& Wellen, R. M. R. (2020). Polypropylene/wood powder/ethylene propylene dienemonomer rubber-maleic anhydride composites: Effect of PPmelt flow index on the thermal, mechanical,thermomechanical, water absorption, and morphologicalparameters. Polymer Composites, 42(1), 484-497.

Ferreira, E. S. S., Luna, C. B. B., Araújo, E. M., Siqueira, D. D., \& Wellen, R. M. R. (2019). Polypropylene/wood powder composites: Evaluation of PP viscosity in thermal, mechanical, thermomechanical, and morphological characters. Journal of Thermoplastic Composite Materials. Epub ahead of print 09 October. https://doi.org/10.1177/0892705719880958.

Giannetti, A. A. M., Agnelli, J. A. M., Lanças, B. Z., Magnabosco, R., Casarin, S. A., \& Bettini, S. H. P. (2012). Lignin as additive in polypropylene/coir composites: Thermal, mechanical and morphological properties. Carbohydrate Polymers, 87(4), 2563-2568.

Hanken, R. B. L., Arimatéia, R. R., Farias, G. M. G., Agrawal, P., Santana, L. N. L., Freitas, D. M. G., \& Mélo, T. J. A. (2019). Effect of natural and expanded vermiculite clays on the properties of eco-friendly biopolyethylene-vermiculite clay biocomposites. Composites Part B: Engineering, $175(10): 107184$.

Immoonen, K., Anttila, U., \& Wikstrom, L. (2019). Coupling of PLA and bleached softwood kraft pulp (BSKP) for enhanced properties of biocomposites. Journal of Thermoplastic Composite Materials, 32(3), 328-341. 
Jesus, L. C. C., Luz, S. M., Leão, R. M., Zattera, A. J., \& Amico, S. C. (2019). Comportamento térmico de compósitos de poliestireno reciclado reforçado com celulose de bagaço de cana. Matéria (Rio J.), 24(3), e-12421.

Kotik, H. G. (2019). Fibras naturais e compósitos reforçados com fibras naturais: a motivação para sua pesquisa e desenvolvimento. Matéria (Rio J.), 24(3), e12477.

Lima, J. C. C., Brandalise, R. N., Almeida, Y. M. B., Melo, T. J. A., \& Vinhas, G. M. (2021). Avaliação das propriedades térmicas de misturas PLA/SEBS com moringa submetidas a degradação em ambiente marinho. Research, Society and Development, 10(4), e12210413249.

Lee, M. C., Koay, S. C., Chan, M. Y., Choo, H. L., Pang, M. M., Chou, P. M., \& Tshai, K. Y. (2020). Properties of poly(lactic acid)/durian husk fiber biocomposites: Effects of fiber content and processing aid. Journal of Thermoplastic Composite Materials, 33(11), 1518-1532.

Luna, C. B. B., Siqueira, D. D., Fereira, E. S. B., Araújo, E. M., \& Wellen, R. M. R. (2019). Reactive compatilization of PCL/WP upon addition of PCL-MA. Smart option for recycling industry. Materials Research Express, 6(12), 125317.

Luna, C. B. C., Silva, D. F., Araújo, E. M., Melo, T. J. A., Bezerra, E. O. T., Siqueira, D. D., \& Oliveira, A. D. (2019b). Blends of polystyrene/shoes waste (SBRr): influence of mixture sequence and compatibilizer. Macromolecular Symposia, 383(1), 1800046.

Luna, C. B. B., Fereira, E. S. B., Siqueira, D. D., Silva, W. A., Araújo, E. M., \& Wellen, R. M. R. (2019c). Tailoring performance of PP/HIPS/SEBS through blending design. Materials Research Express, 6(11), 115321.

Luna, C. B. B., Siqueira, D. D., Fereira, E. S. B., Silva, W. A., Nogueira, J. A. S., \& Araújo, E. M. (2020). From Disposal to Technological Potential: Reuse of Polypropylene Waste from Industrial Containers as a Polystyrene Impact Modifier. Sustainability, 12(13), 5272.

Luna, C. B. B., Silva, D. F., Araújo, E. M., Mélo, T. J. A., \& Oliveira, A. D. (2015). Estudo do comportamento mecânico, termomecânico e morfológico de misturas de poliestireno/composto de borracha reciclada (SBR). Matéria (Rio de Janeiro), 20(2), 322-334.

Marinho, W. S. D., Luna, C. B. B., Araújo, E. M., Lustosa, C. H. A., Filho, C. R. B., \& Duarte, R. N. C. (2020). From disposal to sustainable development: technological potential of poly (lactic acid)(PLA) blends with 3D filament waste. Research, Society and Development, 9(12), e13291210767.

Morais, D. D. S., Siqueira, D. D., Luna, C. B. B., Araújo, E. M., Bezerra, E. B., \& Wellen, R. M. R. (2019). Grafting maleic anhydride onto polycaprolactone: influence of processing. Materials Research Express, 6(5), 055315.

Nguyen, T. C., Ruksakulpiwat, C., \& Ruksakulpiwat, Y. (2020). Effect of cellulose nanofibers from cassava pulp on physical properties of poly(lactic acid) biocomposites. Journal of Thermoplastic Composite Materials, 33(8), 1094-1108.

Poletto, M. (2017). Compósitos termoplásticos com madeira - uma breve revisão. RICA, 2(4), 42-48.

Poletto, M. (2017b). Polypropylene-based wood-plastic composites: Effect of using a coupling agent derived from a renewable resource. Maderas. Ciencia y tecnología, 19(3), $265-272$.

Raia, R. Z., Iwakiri, S., Trianoski, R., Andrade, A. S., \& Kowalski, E. L. (2021). Effects of alkali treatment on modification of the Pinus fibers. Matéria (Rio J.), 26(1), e12936.

Rodrigues, A., Carvalho, B. M., Pinheiro, L. A., Bretas, R. E. S., \& Canevarolo, S. V., Marini, L. (2013). Effect of Compatibilization and Reprocessing on the Isothermal Crystallization Kinetics of Polypropylene/Wood Flour Composites. Polímeros, 23(3), 312-319.

Silva, W. A., Luna, C. B. B., Melo, J. B. C. A., Araújo, E. M., Filho, E. A. S., \& Duarte, R. N. C. (2021). Feasibility of Manufacturing Disposable Cups using PLA/PCL Composites Reinforced with Wood Powder. Journal of Polymers and the Environment, https://doi.org/10.1007/s10924-021-02076-8.

Silva, D. F., Luna, C. B. B., Araújo, E. M., \& Silva, A. L. (2016). Blendas poliméricas: conceitos, obtenção e aplicações. Revista de engenharia e tecnologia, $8(1), 58-77$

Siqueira, D. D., Luna, C. B. B., Fereira, E. S. B., Araújo, E. M., \& Wellen, R. M. R. (2020). Tailored PCL/Macaíba fiber to reach sustainable biocomposites. Journal of Materials Research and Technology, 9(5), 9691-9708.

Siqueira, D. D., Luna, C. B. B., Araújo, E. M., Fereira, E. S. B., \& Wellen, R. M. R. (2019). Biocomposites based on PCL and macaiba fiber. Detailed characterization of main properties. Materials Research Express, 6(9), 095335.

Siqueira, D. D., Luna, C. B. B., Araújo, E. M., Barros, A. B. S., \& Wellen, R. M. R. (2021). Approaches on PCL/macaíba biocomposites - mechanical, thermal, morphological properties and crystallization kinetics. Polymers for Advanced Technologies. https://doi.org/10.1002/pat.5367.

Tarrés, Q., Díaz, D. H., \& Ardanuy, M. (2021). Interface Strength and Fiber Content Influence on Corn Stover Fibers Reinforced Bio-Polyethylene Composites Stiffness. Polymers, 13(5), 768.

Tarrés, Q., Melbo, J. K., Aguilar, M. D., Espinach, F. X., Mutjé, P., \& Carrasco, G. C. (2018). Bio-polyethylene reinforced with thermomechanical pulp fibers: Mechanical and micromechanical characterization and its application in 3D-printing by fused deposition modelling. Composites Part B: Engineering, 153 (11), 70-77.

Tarrés, Q., \& Ardanuy, M. (2020). Evolution of Interfacial Shear Strength and Mean Intrinsic Single Strength in Biobased Composites from Bio-Polyethylene and Thermo-Mechanical Pulp-Corn Stover Fibers. Polymers, 12(6), 1308.

Takemori, M. T. (1979). Towards an understanding of the heat distortion temperature of thermoplastics. Polymer Engineering \& Science, 19(15), 1104-1109. Wang, X., Guo, C., Song, K. (2020). The effects of maleated polybutadiene-grafted polypropylene (MAPB-g-PP) content on the properties of wood flour/polypropylene composites. Journal of Vinyl and Additive Technology, 26(1), 17-23. 
Research, Society and Development, v. 10, n. 7, e23310716277, 2021

(CC BY 4.0) | ISSN 2525-3409 | DOI: http://dx.doi.org/10.33448/rsd-v10i7.16277

Wearn, Y. N., Montagna, L. S., \& Passador, F. R. (2020). Compósitos de fibra de coco/LDPE: efeito do tratamento superficial das fibras de coco em compósitos verdes. Matéria (Rio J.), 25(1), e-12548.

Zahari, W. Z. W., Badri, R. N. R. L., Ardyananta, H., Kurniawan, D., \& Nor, F. M. (2015). Mechanical properties and water absorption behavior of polypropylene/ljuk fiber composite by using silane treatment. Procedia Manufacturing, 2(1), 573-578. 